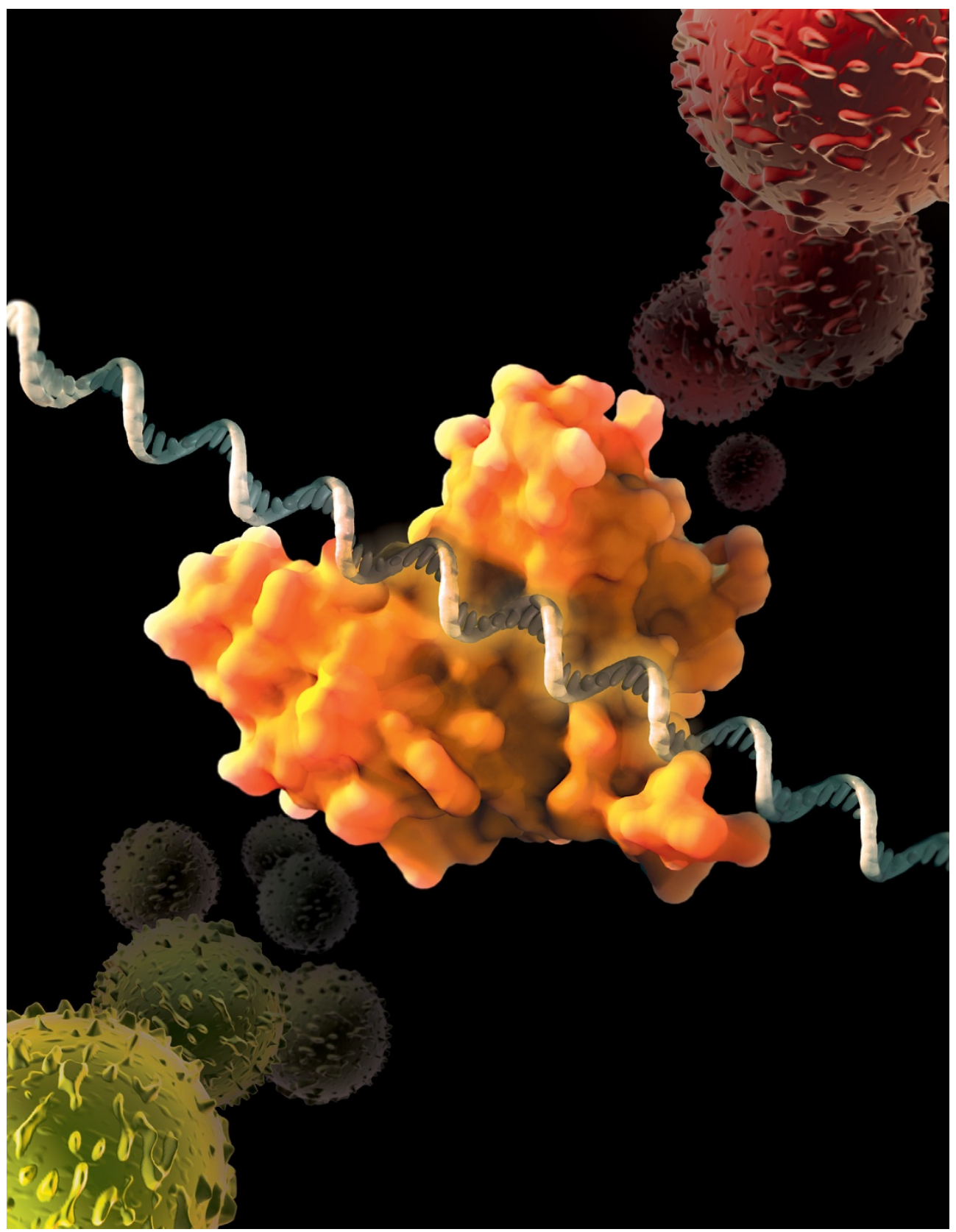

\title{
Emerging complexities of APOBEC3G action on immunity and viral fitness during HIV infection and treatment
}

Monajemi et al. 


\title{
Emerging complexities of APOBEC3G action on immunity and viral fitness during HIV infection and treatment
}

Mahdis Monajemi ${ }^{1 \dagger}$, Claire F Woodworth ${ }^{2 \dagger}$, Jessica Benkaroun ${ }^{2}$, Michael Grant ${ }^{3 *}$ and Mani Larijani ${ }^{3^{*}}$

\begin{abstract}
The enzyme APOBEC3G (A3G) mutates the human immunodeficiency virus (HIV) genome by converting deoxycytidine $(\mathrm{dC})$ to deoxyuridine $(\mathrm{dU})$ on minus strand viral DNA during reverse transcription. A3G restricts viral propagation by degrading or incapacitating the coding ability of the HIV genome. Thus, this enzyme has been perceived as an innate immune barrier to viral replication whilst adaptive immunity responses escalate to effective levels. The discovery of A3G less than a decade ago led to the promise of new anti-viral therapies based on manipulation of its cellular expression and/or activity. The rationale for therapeutic approaches has been solidified by demonstration of the effectiveness of A3G in diminishing viral replication in cell culture systems of HIV infection, reports of its mutational footprint in virions from patients, and recognition of its unusually robust enzymatic potential in biochemical studies in vitro. Despite its effectiveness in various experimental systems, numerous recent studies have shown that the ability of A3G to combat HIV in the physiological setting is severely limited. In fact, it has become apparent that its mutational activity may actually enhance viral fitness by accelerating HIV evolution towards the evasion of both anti-viral drugs and the immune system. This body of work suggests that the role of A3G in HIV infection is more complex than heretofore appreciated and supports the hypothesis that HIV has evolved to exploit the action of this host factor. Here we present an overview of recent data that bring to light historical overestimation of A3G's standing as a strictly anti-viral agent. We discuss the limitations of experimental systems used to assess its activities as well as caveats in data interpretation.
\end{abstract}

\section{The role of APOBEC3G in HIV restriction}

APOBEC3G (A3G) is a recently discovered primate-specific member of the apolipoprotein B mRNA-editing enzyme, catalytic polypeptide-like editing complex family of cytidine deaminase enzymes with potential to inhibit propagation of the human immunodeficiency virus (HIV) $[1,2]$. The APOBEC family includes eleven members in humans: activation-induced cytidine deaminase (AID), APOBEC1, APOBEC2, APOBEC3A-H, and APOBEC4 $[3,4]$. These enzymes convert deoxycytidine $(\mathrm{dC})$ to deoxyuridine (dU) in single stranded DNA (ssDNA) or RNA of human and viral genomes, thereby affecting a variety of physiological functions [5-7]. A3G was discovered through the study of heterokaryons generated between

\footnotetext{
* Correspondence: mgrant@mun.ca; mlarijani@mun.ca

${ }^{\dagger}$ Equal contributors

${ }^{3}$ Division of Biomedical Sciences, Faculty of Medicine, Health Sciences Center, MUN, 300 Prince Phillip Dr., St. John's, NL, A1B 3V6, Canada

Full list of author information is available at the end of the article
}

cells permissive and non-permissive to infection by virion infectivity factor (Vif)-deficient HIV that were used to determine the action of the HIV protein Vif $[1,8,9]$. A3G is primarily expressed in $\mathrm{CD} 4^{+} \mathrm{T}$ lymphocytes, macrophages, and dendritic cells, which are all the natural targets of HIV infection [2,10-14]; although expression in other tissues may be induced by interferon(s) [15-18]. A3G mutates dC in nascent viral minus strand DNA generated by reverse transcription [17-24] and preferentially deaminates $\mathrm{dC}$ in signature trinucleotides (CCE, TCC $)$ often referred to as hotspots [6,19-21]. The resulting dUs can trigger DNA degradation through the action of DNA repair pathways, such as those involving uracil DNA glycosylase and apurinic-apyrimidinic endonuclease $[25,26]$. For viral genomes that evade destruction, the consequent deoxyguanosine (dG) to deoxyadenosine (dA) substitutions in plus strand DNA can alter reading frames, introduce premature translation termination codons, and/or produce mutated viral proteins $[7,20-25]$. In addition, A3G can disrupt propagation

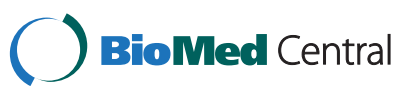


of HIV by binding viral RNA, interfering with the DNA strand transfer acrobatics of reverse transcription, physically blocking reverse transcriptase (RT), and obstructing integration into the host cell genome [24,26-31]. A3G has been shown to block RT activity by decreasing tRNA priming, competing for binding to templates, restricting strand transfer during reverse transcription, and direct binding $[28,32,33]$. Beyond the reverse transcription stage, incorporation of dU into minus strand DNA of the HIV genome has been shown to interfere with synthesis of the complementary plus strand [23]. These findings initially led to the notion that A3G can inhibit viral propagation through pathways dependent or independent of its deamination activity; however, many studies supporting deaminase-independent activities utilized A3G overexpression. It has recently been appreciated that with low level A3G expression, which may be a more accurate representation of the physiological case, deaminase activity is required for viral restriction [34-39]. While the relative contribution of deamination independent activities to viral restriction remains contentious, these may prove more relevant to the action of A3G in restricting endogenous non-long terminal repeat retrotransposons, such as long and short interspersed nuclear elements [40-45]. The anti-retroelement activity of A3G may represent a host strategy to protect its genome from the deleterious effects of transposable elements. A possible mechanism could involve the binding of A3G to retroelements resulting in blockage of their mobility [46].

The recent expansion of a single APOBEC3 gene in mice to seven (APOBEC3A-H) in primates and the relatively high divergence within APOBEC3 enzymes in primates are evidence for immense evolutionary pressure on the locus suggested to possibly be concomitant with the emergence of modern lentiviruses $[3,4,47,48]$. Conversely, the finding that the accelerated rate of A3G divergence predates modern lentiviruses, together with the lack of a clear correlation between human A3G polymorphisms and the progression of acquired immunodeficiency syndrome (AIDS), suggest that lentiviral pressure may be, at best, only partially responsible for expansion of the APOBEC3 locus [49-52]. This manner of growth in host defence capacity can reciprocally drive co-evolution of highly adaptable viruses. In this regard, we highlight an emerging body of evidence suggesting that the activity of A3G may be partially subverted by HIV for its survival benefit. These data support a more complex scenario in which the initial perception of A3G as a strictly anti-viral agent may have been naïve.

\section{Viral and cellular factors limiting APOBEC3G effectiveness} The view of A3G as a potent intrinsic anti-viral factor was largely borne out of findings of high levels of dG to dA hypermutated virus sequences in $\mathrm{di}^{-}$and tri-nucleotide motifs targeted by A3G [53-57]. In stark contrast, the previously recognized mutational machinery of HIV, RT, only introduces approximately one mutation per viral genome during a replication cycle [58]. Supporting the potency of A3G as a mutagenic agent is a wealth of biochemical data showing that it is a highly processive enzyme able to mediate multiple mutations on a given stretch of ssDNA. Accordingly, A3G significantly diminishes viral propagation in several cell culture experimental systems of HIV infection [7,20,22,42,47].

To counteract these activities, lentiviruses have evolved several strategies, primarily in the form of auxiliary proteins such as Vif, which binds and targets newly synthesized A3G for degradation via a ubiquitin-dependent proteosomal pathway [59-69]. A3G is packaged into virions in infected virus-producing cells and it has been shown that it is largely this virion-packaged fraction of A3G rather than the pool of cytoplasmic A3G that is most active on the viral genome in newly infected cells [70-74]. The number of A3G molecules incorporated into each virion is dependent on the level of $A 3 G$ expression in the producer cell [75]. On average, 3 to 11 molecules of A3G are sufficient for effective viral restriction in the target cell [76]. Besides lowering A3G levels through degradation, Vif has also been suggested to directly interfere with A3G encapsidation and may impair its translation [66,74,77-80]. Vif utilizes other co-factors present in the target cell to ubiquitinate $\mathrm{A} 3 \mathrm{G}$ and it was recently shown that Core binding factor (CBF)- $\beta$, a cellular transcription factor, is required for Vif-mediated degradation of A3G [81,82]. As a result, when Vif is present, the mutation levels induced by A3G and its effectiveness in viral restriction are diminished. That Vif is essential for HIV replication in A3Gexpressing cells, and that the sole function of Vif was thought to be A3G inactivation, lent credence to the notion that $\mathrm{A} 3 \mathrm{G}$ is a potent restrictor of $\mathrm{HIV}$ propagation [83]. On the other hand, it is now appreciated that even in the presence of Vif, A3G can still cause sub-lethal levels of $\mathrm{dG}$ to dA mutations $[19,84]$. It is possible that the preferential targeting of newly synthesized A3G by Vif leaves a fraction of previously synthesized A3G intact [85]. In addition, it appears that Vif expression does not completely abolish A3G activity and the correlation between the levels of viral infectivity and A3G inhibition by Vif is not absolute [62,78]. Other functions for Vif and Vifmediated ubiquitination, besides A3G degradation, are also coming to light. For instance, along with the auxiliary protein Vpr, Vif can induce G2 cell-cycle arrest, which may contribute to $\mathrm{CD} 4^{+} \mathrm{T}$ lymphocyte depletion [86-89]. Vif thus mediates several functions that are independent of its interaction with A3G and is a variable negative regulator of A3G activity rather than a complete inhibitor.

A3G action is further limited by its entrapment in high-molecular-mass ribonuclear complexes (HMM) that may reach megadaltons in size, mediated by non-specific 
binding of cellular and/or viral RNA and proteins [12,71,90-97]. The shuttling of A3G into newly synthesized virions depends on binding viral RNA and/or proteins [98-101]. The requirement for high affinity interactions with RNA/DNA substrates may explain the evolution of A3G (and other APOBEC enzymes, e.g. AID) to contain an unusually high number of charged residues on its surface [102-104]. Ironically, this same attribute necessary to enact the anti-viral function of A3G may also be a key contributor to limiting its antiviral function through HMM formation. Reversion of HMM to low-molecular-mass (LMM) A3G can be experimentally mediated by treatment with $\mathrm{RNase} A / \mathrm{H}$ $[70,71,105]$. The RNase $\mathrm{H}$ activity of RT is thought to release viral RNA-bound A3G, allowing it to act on the proximal minus strand DNA during its synthesis $[2,19,75]$. Enzymatically active A3G able to be incorporate into newly synthesized virions is strictly found outside of the HMM complexes in the LMM fraction $[73,106]$. The LMM form primarily resides in peripheral blood-derived resting $\mathrm{CD} 4^{+} \mathrm{T}$ cells and monocytes [12]; however, upon activation of $\mathrm{CD}^{+}{ }^{+} \mathrm{T}$ cells or differentiation of monocytes into macrophages, a higher proportion of A3G is shuttled to HMM complexes [2,91]. Although this was suggested to be a mechanism that restricted the infection of resting $\mathrm{T}$ cells by HIV, subsequent knockout studies of LMM A3G in resting $\mathrm{CD} 4^{+} \mathrm{T}$ cells did not render these cells permissive to HIV infection, thus indicating that the difference in the LMMversus HMM-bound proportion of A3G is not the sole mechanism for resistance of resting $\mathrm{CD}^{+}{ }^{+} \mathrm{T}$ cells to HIV infection $[107,108]$. Beyond the induction of HMM formation by HIV through cellular activation processes, Vif has been shown to directly promote HMM production [109]. Remarkably complex co-evolution is evident considering the intimate linkage between HIV infection and HMM formation and the notable level of mechanistic integration between A3G function and the viral replication machinery. The RNase $\mathrm{H}$ activity of RT is at once both necessary and detrimental to viral propagation due to its role in the release of active A3G.

The complexities surrounding regulation of Vif activity and HMM formation notwithstanding, it is clear that both result in diminished A3G efficacy. It is possible that mutations introduced by A3G only succeed in restricting viral replication at a sub-optimal level and conversely may assist the virus by generating sequence variation $[35,39,84]$. Consequently, an alternative view that A3G activity can contribute to viral fitness has recently gained strong support. In the following sections, we highlight evidence for the pro-viral activities of A3G. At the same time, we discuss caveats of experimental systems and data interpretation that must henceforth be considered in development of a revised and better-informed picture of A3G function.

\section{The role of APOBEC3G in generation of anti-viral drug resistant HIV}

Gain of resistance to drug(s) used in the treatment of HIV is a major determinant of viral evolution during the course of disease. To date, almost a hundred drug resistance mutation sites have been identified in the HIV genome [110]. These induce resistance to common anti-HIV drugs acting as nucleoside/nucleotide analogue RT inhibitors, such as 2',3'-dideoxy-3'-thiacytidine (3TC), abacavir $(\mathrm{ABC})$, and 2',3'-dideoxyinosine (DDI), as well as non-nucleoside/nucleotide analogue RT inhibitors, including Nevirapine (NVP), Delavirdine (DLV), and Efavirenz (EFV) [110]. Drug resistance mutations function directly by altering drug targets or indirectly by modifying pathways that contribute to drug escape. Many drug resistance mutations have been shown to reside in A3G hotspots [111].

A bioinformatics study assessed the probability of A3G mutations in known drug resistance sites taking into consideration the double-crested gradient of A3G-induced mutational levels throughout the HIV genome. Out of $52,000 \mathrm{G}$ to A mutations, only 695 (1.3\%) were located in drug resistance sites [112]. In this context, the investigators reported a modest correlation between A3G activity and the generation of drug resistance mutations relative to the overall footprint of A3G on the HIV genome [19,112,113]; however, recent experimental evidence more strongly implicates A3G in the generation of drug resistance mutations. For example, the very common M184I(V) mutation of RT that causes resistance to 3TC and, to a lesser extent, $\mathrm{ABC}$ and DDI, is located in an A3G hotspot (TCCAT to TCUAT) and is produced by A3G in vitro during HIV replication in cell culture systems [114]. Intriguingly, this was observed in the absence of 3TC in as many as $40 \%$ of sequenced proviruses, reflecting a pre-treatment pool of resistant viruses poised for propagation after drug exposure [29,115-118]. Because this mutation may in fact reduce viral replication fitness in the absence of 3TC [119-122], it is likely that this measurement actually underestimates the role of A3G in the generation of this mutation. In support of this notion, the M184I mutation emerges at significantly higher rates when the virus is grown in A3G-expressing as compared to A3G non-expressing host cells, indicating that A3G activity is the major source of this mutation [123]. This is a striking example of the parallel role of A3G in simultaneously aiding host and virus: in the same manner that it acts as a pre-existing innate immune factor that fortifies host defenses prior to viral exposure, A3G boosts the inherent ability of HIV to gain resistance even before drug treatment. That this mutation is associated with a decline in viral fitness may indicate that drug resistance presents a significant source of pressure in viral evolution resulting in the gain or maintenance of A3G hotspots in key positions in the viral genome. 
If the contribution of A3G action to drug resistance and survival of HIV is a biologically considerable one, the evolution of HIV during disease could involve active relaxation of A3G inhibition. Indeed, direct evidence for this phenomenon was provided by the prevalence of the Vif $\mathrm{K} 22 \mathrm{H}$ mutation in patients failing drug treatment, as compared to treatment-naïve patients [124,125]. Vif K22 is a key residue for interaction with A3G, and Vif $\mathrm{K} 22 \mathrm{H}$ exhibits reduced effectiveness in neutralizing A3G [115]. Ex vivo infection of peripheral blood mononuclear cells (PBMCs) with viral stocks harboring various other Vif mutations that are unable to deactivate A3G (e.g. Vif $\mathrm{K} 22 \mathrm{E}$ ) yielded a significant increase in the generation of M184I mutants [114]. In addition, several drug resistance mutations, including M184I in RT and G16E/M36I in the protease, are significantly more common in patients harboring elevated relative levels of $\mathrm{K} 22 \mathrm{H}$-mutated viruses [125]. Like the M184I mutation, both G16E and M36I mutation sites are located in A3G hotspots. Thus, not only does HIV benefit from spontaneous pre-drug treatment A3G-induced mutations in a passive, somewhat random manner, it appears that resistance sites for some of the most commonly used drugs arose in A3G hotspots. This in no way implies viral sentience, but merely indicates a selective advantage derived from the overlap of sites more susceptible to mutation (A3G hotspots) being able to confer drug resistance.

\section{The contribution of APOBEC3G to the evasion of adaptive immunity by HIV}

Restrictions imposed on the activity of A3G by Vif and HMM limit its effectiveness as an innate immune agent; however, following the first weeks of HIV infection, development of B and $\mathrm{T}$ cell mediated adaptive immunity partially controls viremia [116-118,126]. A central facet of the adaptive immune response is elimination of infected target cells by cytotoxic $\mathrm{T}$ cells $(\mathrm{CTL})$, as highlighted by the close inverse association between robustness of the CTL response with viremia levels and disease progression [127-129]. Thus, evasion of the CTL response is thought to be a powerful driving force for the evolution of HIV during disease, as confirmed by several studies showing the prevalence of CTL escape in HIV infection $[130,131]$. CTL evasion may result from alterations in CTL access to infected cells. For instance, the auxiliary HIV protein Nef modulates class I MHC expression to decrease the recognition and killing of infected cells [132]. Alternatively, CTL evasion may result from alterations in the interactions between the CTL and infected target cell. Mutations in CTL recognition epitopes have been shown to mediate CTL evasion through modulating the efficacy of CTL activation [133-135].

As in the case of drug resistance, it is possible that HIV can exploit the limited non-lethal action of A3G to generate CTL escape mutants. In support of this model, a study examining CTL escape during early infection found that approximately a third of the rapidly mutating sites mediating CTL escape were embedded in A3G hotspots, with more highly mutating sites being relatively enriched in A3G hotspots [136]. Twenty-four rapidly diversifying sites were identified at which $G$ to $A$ mutations were 2-3 fold more frequent than the overall $G$ to A mutation rate across the entire HIV genome (29 versus $12 \%)$. Fourteen of these sites located in or near CTL epitopes. These data suggest that it may be advantageous towards immune escape for HIV to maintain A3G hotspots in areas where mutations can affect processing, presentation or recognition of $\mathrm{T}$ cell epitopes, or conversely to establish $\mathrm{T}$ cell epitopes near A3G hotspots.

In contrast, another study reported that A3G mutations enhance the virus-specific CTL response through the introduction of premature stop codons into the HIV genome that cause the generation of truncated or misfolded proteins [137]. In this study, Vif+ or Vif- HIV was produced in the presence or absence of A3G in a cell line and subsequently used to infect PBMCs followed by assessing their susceptibility to MHC-matched peptidespecific CTL clones. It is possible that the finding of enhanced target cell killing as a result of A3G activity reflects an inherent bias of the specificity of the CTL clones examined. In addition, given the numbers, diversity, and relative scarcity of CTL specific for each particular peptide in vivo, the general biological relevance of this work remains to be determined. Therefore, although A3G appears to play a role beyond innate immunity and modulate adaptive immunity, further work is required to elucidate the nature and extent of this activity.

\section{Manipulation of APOBEC3G effectiveness: implications and challenges for the design of therapeutic approaches}

To date, multiple avenues have been suggested and/or pursued towards exploitation of A3G as an antiviral therapy. These approaches include the development of small molecules that inhibit the interaction between Vif and A3G and/or inhibit interactions with cellular factors that act downstream of Vif, enhancement of LMM formation over HMM formation, and increasing A3G levels by treatment with interferons or gene-therapy delivery of A3G along with the restriction factor Trim $5 \alpha$ [70,138-140].

Strategies to down-regulate the action of Vif and HMM that were initially suggested as therapeutic approaches have recently been questioned in light of the increasingly apparent pro-viral activities of A3G [35,141]. Disturbing the Vif-APOBEC interaction presents a delicate challenge because subtle adjustments to Vif activity have been shown to modulate levels of A3G activity. For instance, naturally occurring patient-derived virions harboring Vif mutations selectively exhibit viral genome 
sequence variations consistent with survival advantage under their environmental pressures [25,54,125]. Incomplete Vif inhibition might increase effective A3G concentrations and in so doing actually accelerate viral evolution by only modestly increasing non-lethal mutation rates $[22,111]$. On the other hand, complete Vif inhibition may result in A3G activity levels high enough to tip the balance towards immunity through mutation loads capable of disabling viral replication. The isolation of viral sequences harboring Vif mutants significantly diminished in their ability to neutralize A3G challenges this scenario as it brings into question the ability of A3G to fully abrogate the propagation of Vif-deficient viruses [124,125]. Furthermore, it may also be important to consider the involvement of Vif inactivation in the generation of drug resistance as a cautionary note against therapies designed for complete elimination of Vif activity.

Studies of the effect of A3G expression levels on HIV disease progression rates in both humans and other primates have yielded conflicting results. One investigation reported an inverse correlation between A3G expression levels and disease progression [142,143], while another noted no such association [144]. A third study conducted on SIV-infected rhesus macaques reported an inverse correlation between A3G expression levels and disease progression [145]. Further work will be required to conclusively define any association between A3G expression patterns or levels and HIV disease progression. In addition, it is not clear whether A3G expression levels can influence the relative extent of its pro- versus anti-viral activities. If indeed there is any correlation, it remains to be determined where the threshold level of A3G activity lies and whether it varies during the course of infection. Whatever the pivotal point, the underlying premise that regulating A3G activity by modulating Vif/HMMs can alter viral mutation levels to the detriment of HIV may be flawed in viewing HIV as an acquiescent canvas for mutational activity. Examination of the spectrum of A3G-induced mutations during the viral life cycle paints a different picture in which there is a high level of mutation in viral DNA, an intermediate level in cellular RNA, and a low level in viral RNA. Non-advantageous or detrimental mutations are serially filtered out during the transcription, nuclear-cytoplasmic transport, translation and assembly phases of the viral life cycle, resulting in a pool of virions emerging from the host cell that bear a suppressed footprint of total A3G mutational activity - a process termed purifying selection [146]. Although at first glance it ought to decrease viral variation, purifying selection is balanced in favour of HIV by other diversification processes, such as recombination between mutated and wild-type viral genomes [147,148]. This is a sophisticated mechanism of protection for the virus as it enhances the potential for beneficial mutations to propagate quickly and represents a heretofore unappreciated layer of complexity when considering therapeutic strategies centered around modulating the activity and/or levels of A3G.

\section{The relative contribution of APOBEC $3 \mathrm{G}$ in the context of other viral factors to HIV evolution}

Formulating therapeutic strategies also requires a careful assessment of the relative contributions of non-A3G factors to the sequence variation of HIV. In general, retroviral genomes are prone to a high frequency of mutation [149-153]. The elevated error rate of the HIV RT, alterations in the nucleoside triphosphate (NTP) and deoxynucleotide triphosphate (dNTP) levels that affect polymerase accuracies, and the lack of proofreading machinery during viral genome replication all contribute to the highly mutagenic nature of viral genomes [58,151]. In addition to mutations, HIV exhibits a notably high rate of genomic recombination amongst retroviruses, possibly due to its cellular transmission properties resulting in frequent co-infection by genetic variants [154157]. Unlike in humans, recombination in retroviruses does not result from breakage and rejoining of DNA, but is instead mediated by the ability of RT to switch templates between the two encapsidated proviral RNAs [158-160].

Distinguishing between the actions of RT versus A3G is essential in determining the relative contribution of each to HIV pathogenesis given that its genome is predominated by $\mathrm{dA}$ nucleotides, and $\mathrm{dG}$ to $\mathrm{dA}$ changes are key to the generation of many drug resistance variants $[111,161,162]$. Prior to the discovery of A3G, RT was viewed as the main generator of genetic diversification in the HIV genome throughout the course of infection; however, both RT and A3G most frequently induce dG to $\mathrm{dA}$ transition mutations on the plus viral DNA strand [163]. Although a degree of uncertainty arises in assigning the source of hypermutations in the HIV genome, the fact that $\mathrm{A} 3 \mathrm{G}$ preferentially deaminates $\mathrm{dC}$ nucleotides in signature trinucleotide hotspots (CCE, TCCE) can be used to assign mutations [6,19-21]. In contrast to RT, which is capable of introducing one to two mutations in each viral genome per replication cycle [58], A3G is a highly processive and robust deaminase enzyme [164]. The rate of dG to dA hypermutation found in HIV genomes is approximately 1000 fold higher than RT alone would be expected to introduce [71]. Furthermore, A3Gexpressing cells support significantly more HIV hypermutation than their A3G-deficient counterparts. While the impact of A3G on mutational load is tempered during wild-type HIV infection by factors such as Vif and HMM, and potentially obscured from the circulating virus pool by purifying selection, these and other observations provide evidence that $\mathrm{A} 3 \mathrm{G}$ can and does make substantial contributions to HIV sequence variation 


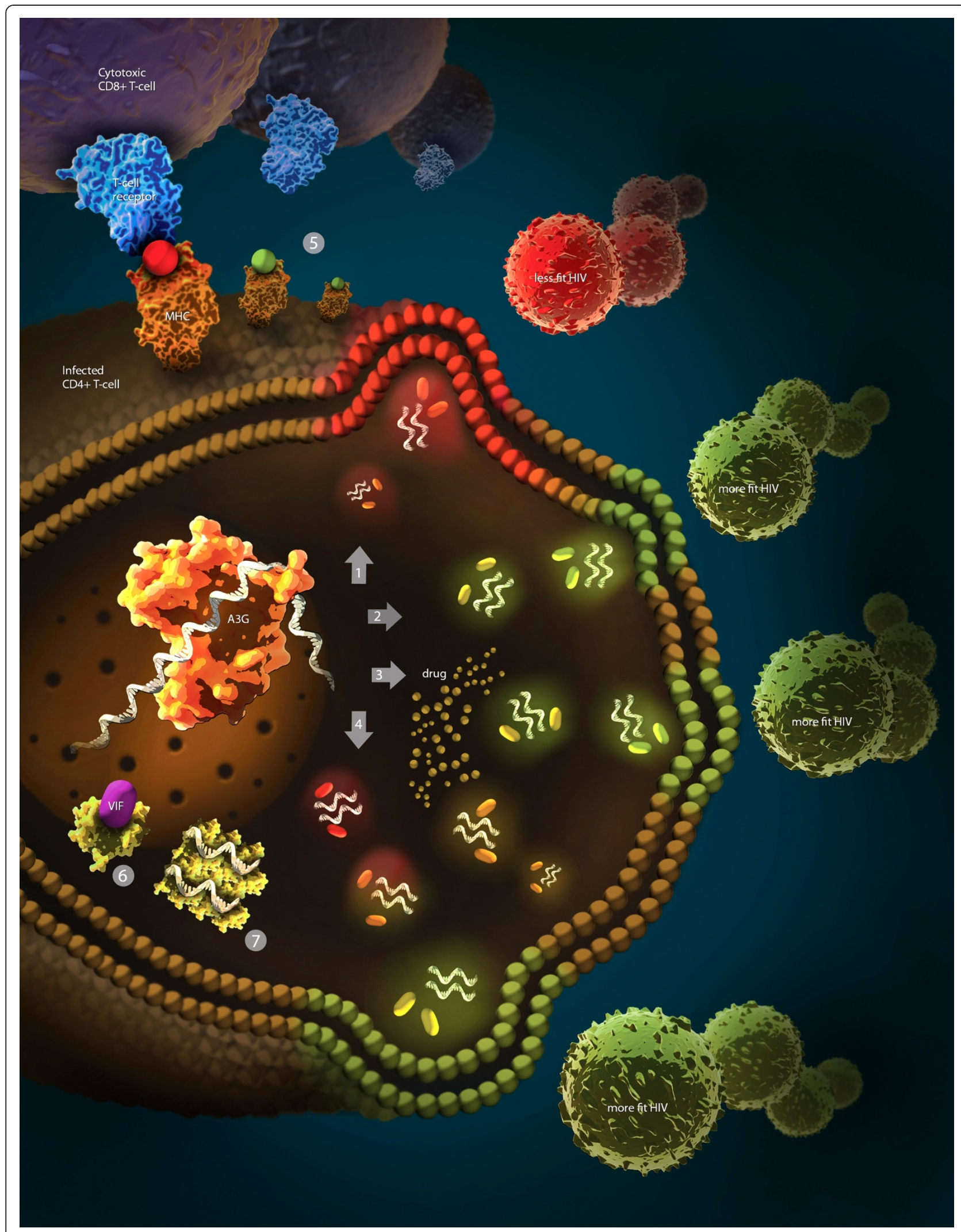

Figure 1 (See legend on next page.) 


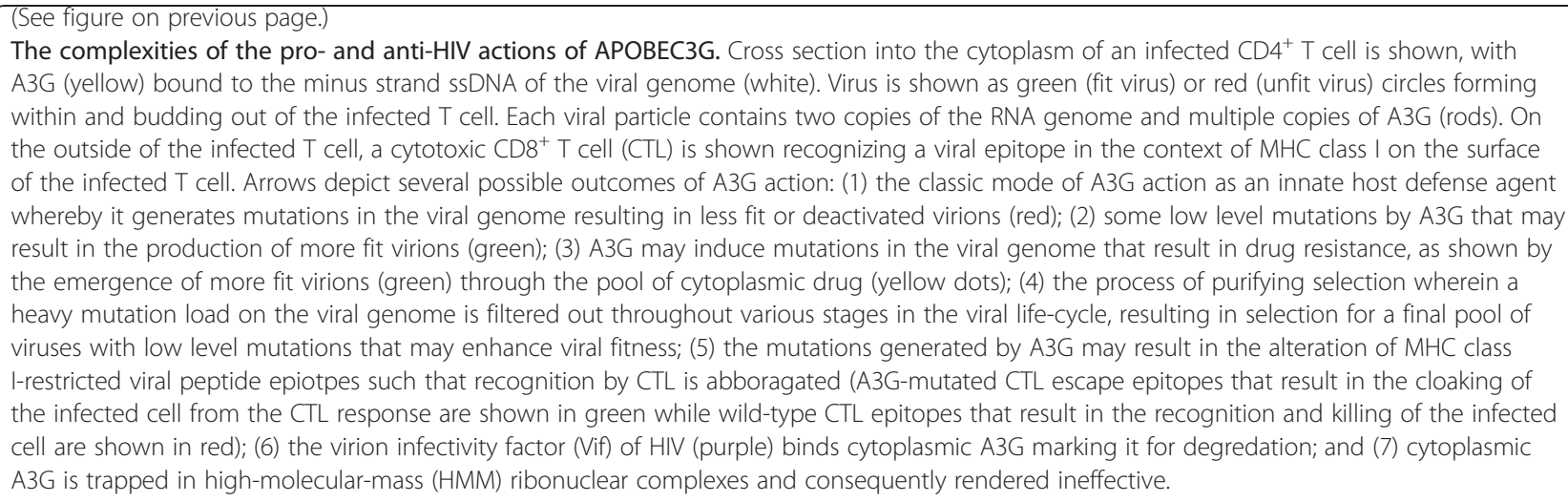

[123]. Somewhere between the unfettered A3G activity that causes a lethal mutational load and complete A3G inhibition by Vif and other cellular factors lies a level of activity with the potential to favor drug resistance, immune escape and viral fitness. Given the demonstrated ability of HIV to adapt to its host, it would be surprising if adaptations deriving benefit from some level of A3G activity have not occurred.

\section{Conclusions}

Figure 1 illustrates the various topics discussed herein with respect to the dual role of A3G in aiding the host or virus. As shown, there are clear instances when HIV can take advantage of A3G-induced mutation across a range of activity levels. Low mutation rates do not inactivate the viral genome and may in fact contribute to both drug resistance and immune escape. Conversely, HIV genomes suffering high mutation rates may be filtered out during viral replication to favor viral progeny with better fitness. Therefore, an ominous picture emerges wherein regardless of the action of A3G, HIV gradually gains the upper hand as a result of its fast replication rate and purifying selection processes that allow it to essentially optimize A3G mutation loads in progeny virions and better adapt to host defences and other selective pressures.

On the other hand, interpretation of studies examining the effect of A3G on the drug and CTL escape mutations in the HIV genome is subject to a major caveat. To date, studies identifying CTL escape or drug resistance mutations have been conducted using two general approaches: firstly, by searching for such mutations in clinical isolates; and secondly, by analyzing mutations in cell culture infection systems where A3G is expressed. We suggest that these types of studies are inherently biased towards generating the observed results and missing the bigger picture. In the first case, the virus pool obtained from infected individuals will inevitably be enriched for CTL and drug escape mutants as these have a replication advantage wherein virions harboring CTL or drug target motifs modified by A3G in a way that supports the opposite outcome (i.e. enhanced CTL recognition or increased drug susceptibility) would have been efficiently eliminated. Therefore, A3G could potentially create new or more immunogenic epitopes that have not yet been characterized. Furthermore, any suggestion that CTL escape is merely serendipitous neglects the point that a limited number of high quality escape epitopes are selected, as opposed to a large quantity of epitopes with low immune evading potential or substantial negative effects on viral fitness. HIV features a very economical propagation process in that the cost of having some genes manipulated is in-turn compensated for by a net effect favoring evasion of composite selective pressure. In the case of cell culture systems examining the role of A3G in generating drug resistance variants, the same caveat stands. That is, multiple drug resistance mutations have been identified and well characterized due to their prominence in patients. A3Ginduced mutations that may conversely enhance drug susceptibility have not been identified because of their scarcity caused by more rapid elimination. Accordingly, we suggest that instances where A3G may in fact bestow the upper hand upon the host by generating mutations that enhance CTL recognition or drug susceptibility have likely been underestimated because of their inevitable transience. It is probable that the pro- and anti-viral activities of A3G are not mutually exclusive and that, at different points throughout HIV infection and in different patients, both scenarios unfold; however, the principles of purifying selection are active at the level of individual cells and that of the entire host organism, which buries the evidence of maladaptive A3Gimposed mutations beneath an avalanche of fast replicating adapted variants. While new experimental approaches are required to identify the relative proportion of both categories of A3G-induced mutations in an unbiased manner, the final outcome following multiple selection processes will determine the global impact of A3G mutations. Even if thousands of A3G-induced mutations favoring the host occur for a single mutation that favors HIV, the net advantage will be to HIV as long as one favorable mutation becomes incorporated into the circulating viral pool. Thus, the overall context within which A3G acts is probably just 
as relevant as the ratio of pro- versus anti-viral mutations. Resolution of this bigger picture will be critical in order to guide future therapeutic strategies centered on altering A3G activity.

\section{Competing interests}

Authors do not have any competing interests.

\section{Acknowledgments}

ML is supported by a CIHR New Investigator award and a CIHR operating grant (MOP111132). MG is supported by CIHR operating grants (HOP-111725, HOP-93428) and catalyst grant (HC1-112568). Figure 1 was designed by Ms. Enid Hajderi, MSCBMC, and ML. We are grateful to Ms. Enid Hajderi for creating the cover artwork and artwork for Figure 1.

\section{Author details}

${ }^{1}$ Immunology and Infectious Diseases Program, Division of Biomedical Sciences, Faculty of Medicine, Memorial University of Newfoundland, Newfoundland, Canada. ${ }^{2}$ Mani Larijani, Division of Biomedical Sciences, Faculty of Medicine, Health Sciences Center, MUN, 300 Prince Phillip Dr., St. John's, NL, A1B 3V6, Canada. 'Division of Biomedical Sciences, Faculty of Medicine, Health Sciences Center, MUN, 300 Prince Phillip Dr., St. John's, NL, A1B 3V6, Canada.

\section{Authors' contributions}

MM and CW analyzed the literature and drafted the manuscript. JB contributed to the introduction. MG and ML prepared and edited the manuscript. All authors read and approved the final manuscript.

Received: 3 February 2012 Accepted: 30 April 2012

Published: 30 April 2012

\section{References}

1. Sheehy AM, Gaddis NC, Choi JD, Malim MH: Isolation of a human gene that inhibits HIV-1 infection and is suppressed by the viral Vif protein. Nature 2002, 418:646-650.

2. Chiu YL, Soros VB, Kreisberg JF, Stopak K, Yonemoto W, Greene WC: Cellular APOBEC3G restricts HIV-1 infection in resting CD4+ T cells. Nature 2005, 435:108-114.

3. Conticello SG, Thomas CJ, Petersen-Mahrt SK, Neuberger MS: Evolution of the AID/APOBEC family of polynucleotide (deoxy)cytidine deaminases. Mol Biol Evol 2005, 22:367-377.

4. Jarmuz A, Chester A, Bayliss J, Gisbourne J, Dunham I, Scott J, Navaratnam $\mathrm{N}$ : An anthropoid-specific locus of orphan C to U RNA-editing enzymes on chromosome 22. Genomics 2002, 79:285-296.

5. Conticello SG: The AID/APOBEC family of nucleic acid mutators. Genome Biol 2008, 9:229.

6. Beale RC, Petersen-Mahrt SK, Watt IN, Harris RS, Rada C, Neuberger MS: Comparison of the differential context-dependence of DNA deamination by APOBEC enzymes: correlation with mutation spectra in vivo. $J \mathrm{Mol}$ Biol 2004, 337:585-596.

7. Harris RS, Bishop KN, Sheehy AM, Craig HM, Petersen-Mahrt SK, Watt IN, Neuberger MS, Malim MH: DNA deamination mediates innate immunity to retroviral infection. Cell 2003, 113:803-809.

8. Simon JH, Gaddis NC, Fouchier RA, Malim MH: Evidence for a newly discovered cellular anti-HIV-1 phenotype. Nat Med 1998, 4:1397-1400.

9. Madani N, Kabat D: An endogenous inhibitor of human immunodeficiency virus in human lymphocytes is overcome by the viral Vif protein. J Virol 1998, 72:10251-10255.

10. Peng G, Greenwell-Wild T, Nares S, Jin W, Lei KJ, Rangel ZG, Munson PJ, Wahl SM: Myeloid differentiation and susceptibility to HIV-1 are linked to APOBEC3 expression. Blood 2007, 110:393-400.

11. Pido-Lopez J, Whittall T, Wang Y, Bergmeier LA, Babaahmady K, Singh M, Lehner T: Stimulation of cell surface CCR5 and CD40 molecules by their ligands or by HSP70 up-regulates APOBEC3G expression in CD4(+) T cells and dendritic cells. J Immunol 2007, 178:1671-1679.

12. Stopak KS, Chiu YL, Kropp J, Grant RM, Greene WC: Distinct patterns of cytokine regulation of $A P O B E C 3 G$ expression and activity in primary lymphocytes, macrophages, and dendritic cells. J Biol Chem 2007, 282:3539-3546.
13. Koning FA, Newman EN, Kim EY, Kunstman KJ, Wolinsky SM, Malim MH: Defining APOBEC3 expression patterns in human tissues and hematopoietic cell subsets. J Virol 2009, 83:9474-9485

14. Pion M, Granelli-Piperno A, Mangeat B, Stalder R, Correa R, Steinman RM, Piguet $\mathrm{V}$ : APOBEC3G/3 F mediates intrinsic resistance of monocytederived dendritic cells to HIV-1 infection. J Exp Med 2006, 203:2887-2893.

15. Bonvin M, Achermann F, Greeve I, Stroka D, Keogh A, Inderbitzin D, Candinas D, Sommer P, Wain-Hobson S, Vartanian JP, Greeve J: Interferoninducible expression of APOBEC3 editing enzymes in human hepatocytes and inhibition of hepatitis $B$ virus replication. Hepatology 2006, 43:1364-1374

16. Komohara Y, Suekane S, Noguchi M, Matsuoka K, Yamada A, Itoh K: Expression of APOBEC3G in kidney cells. Tissue Antigens 2007, 69:95-98.

17. Peng G, Lei K, Jin W, Greenwell-Wild T, Wahl SM: Induction of APOBEC3 family proteins, a defensive maneuver underlying interferon-induced anti-HIV-1 activity. J Exp Med 2006, 203:41-46.

18. Tanaka $Y$, Marusawa $H$, Seno $H$, Matsumoto $Y$, Ueda $Y$, Kodama $Y$, Endo $Y$, Yamauchi J, Matsumoto T, Takaori-Kondo A, et al: Anti-viral protein APOBEC3G is induced by interferon-alpha stimulation in human hepatocytes. Biochem Biophys Res Commun 2006, 341:314-319.

19. Yu Q, Konig R, Pillai S, Chiles K, Kearney M, Palmer S, Richman D, Coffin JM, Landau NR: Single-strand specificity of APOBEC3G accounts for minusstrand deamination of the HIV genome. Nat Struct Mol Biol 2004, 11:435-442.

20. Lecossier D, Bouchonnet F, Clavel F, Hance AJ: Hypermutation of HIV-1 DNA in the absence of the Vif protein. Science 2003, 300:1112.

21. Mangeat B, Turelli P, Caron G, Friedli M, Perrin L, Trono D: Broad antiretroviral defence by human APOBEC3G through lethal editing of nascent reverse transcripts. Nature 2003, 424:99-103.

22. Zhang H, Yang B, Pomerantz RJ, Zhang C, Arunachalam SC, Gao L: The cytidine deaminase CEM15 induces hypermutation in newly synthesized HIV-1 DNA. Nature 2003, 424:94-98.

23. Klarmann GJ, Chen X, North TW, Preston BD: Incorporation of uracil into minus strand DNA affects the specificity of plus strand synthesis initiation during lentiviral reverse transcription. J Biol Chem 2003, 278:7902-7909.

24. Mbisa JL, Barr R, Thomas JA, Vandegraaff N, Dorweiler IJ, Svarovskaia ES, Brown WL, Mansky LM, Gorelick RJ, Harris RS, et al: Human immunodeficiency virus type $1 \mathrm{cDNAs}$ produced in the presence of APOBEC $3 G$ exhibit defects in plus-strand DNA transfer and integration. J Virol 2007, 81:7099-7110.

25. Simon V, Zennou V, Murray D, Huang Y, Ho DD, Bieniasz PD: Natural variation in Vif: differential impact on $A P O B E C 3 G / 3 \mathrm{~F}$ and a potential role in HIV-1 diversification. PLoS Pathog 2005, 1:e6.

26. Newman EN, Holmes RK, Craig HM, Klein KC, Lingappa JR, Malim MH, Sheehy AM: Antiviral function of APOBEC3G can be dissociated from cytidine deaminase activity. Curr Biol 2005, 15:166-170.

27. Anderson JL, Hope TJ: APOBEC3G restricts early HIV-1 replication in the cytoplasm of target cells. Virology 2008, 375:1-12.

28. Li XY, Guo F, Zhang L, Kleiman L, Cen S: APOBEC3G inhibits DNA strand transfer during HIV-1 reverse transcription. J Biol Chem 2007, 282:32065-32074.

29. Navarro F, Bollman B, Chen H, Konig R, Yu Q, Chiles K, Landau NR: Complementary function of the two catalytic domains of APOBEC3G. Virology 2005, 333:374-386.

30. Luo K, Wang T, Liu B, Tian C, Xiao Z, Kappes J, Yu XF: Cytidine deaminases APOBEC3G and APOBEC3F interact with human immunodeficiency virus type 1 integrase and inhibit proviral DNA formation. J Virol 2007, 81:7238-7248.

31. Mbisa JL, Bu W, Pathak VK: APOBEC3F and APOBEC3G inhibit HIV-1 DNA integration by different mechanisms. J Virol 2010, 84:5250-5259.

32. Guo F, Cen S, Niu M, Yang Y, Gorelick RJ, Kleiman L: The interaction of APOBEC3G with human immunodeficiency virus type 1 nucleocapsid inhibits tRNA3Lys annealing to viral RNA. J Virol 2007, 81:11322-11331.

33. Wang X, Ao Z, Chen L, Kobinger G, Peng J, Yao X: The Cellular Antiviral Protein APOBEC3G Interacts with HIV-1 Reverse Transcriptase and Inhibits Its Function during Viral Replication. J Virol 2012, 86:3777-3786.

34. Browne EP, Allers C, Landau NR: Restriction of HIV-1 by APOBEC3G is cytidine deaminase-dependent. Virology 2009, 387:313-321.

35. Smith HC: APOBEC3G: a double agent in defense. Trends Biochem Sci 2011, 36:239-244

36. Miyagi E, Opi S, Takeuchi H, Khan M, Goila-Gaur R, Kao S, Strebel K: Enzymatically active APOBEC3G is required for efficient inhibition of human immunodeficiency virus type 1. J Virol 2007, 81:13346-13353. 
37. Schumacher AJ, Nissley DV, Harris RS: APOBEC3G hypermutates genomic DNA and inhibits Ty1 retrotransposition in yeast. Proc Natl Acad Sci U S A 2005, 102:9854-9859.

38. Schumacher AJ, Hache G, Macduff DA, Brown WL, Harris RS: The DNA deaminase activity of human APOBEC3G is required for Ty1, MusD, and human immunodeficiency virus type 1 restriction. J Virol 2008, 82:2652-2660.

39. Holmes RK, Koning FA, Bishop KN, Malim MH: APOBEC3F can inhibit the accumulation of HIV-1 reverse transcription products in the absence of hypermutation. Comparisons with APOBEC3G. J Biol Chem 2007, 282 (4):2587-2595. Epub 2006 Nov 22

40. Stenglein MD, Harris RS: APOBEC3B and APOBEC3F inhibit L1 retrotransposition by a DNA deamination-independent mechanism. J Biol Chem 2006, 281:16837-16841.

41. Muckenfuss H, Hamdorf M, Held U, Perkovic M, Lower J, Cichutek K, Flory E, Schumann GG, Munk C: APOBEC3 proteins inhibit human LINE-1 retrotransposition. J Biol Chem 2006, 281:22161-22172.

42. Turelli $P$, Mangeat $B$, Jost $S$, Vianin S, Trono D: Inhibition of hepatitis B virus replication by APOBEC3G. Science 1829, 2004:303.

43. Bogerd HP, Wiegand HL, Hulme AE, Garcia-Perez JL, O'Shea KS, Moran JV Cullen BR: Cellular inhibitors of long interspersed element 1 and Alu retrotransposition. Proc Natl Acad Sci U S A 2006, 103:8780-8785.

44. Esnault C, Heidmann O, Delebecque F, Dewannieux M, Ribet D, Hance AJ, Heidmann T, Schwartz O: APOBEC3G cytidine deaminase inhibits retrotransposition of endogenous retroviruses. Nature 2005, 433:430433

45. Esnault C, Millet J, Schwartz O, Heidmann T: Dual inhibitory effects of APOBEC family proteins on retrotransposition of mammalian endogenous retroviruses. Nucleic Acids Res 2006, 34:1522-1531.

46. Chiu YL, Witkowska HE, Hall SC, Santiago M, Soros VB, Esnault C, Heidmann T, Greene WC: High-molecular-mass APOBEC3G complexes restrict Alu retrotransposition. Proc Natl Acad Sci U S A 2006, 103:15588-15593.

47. Zhang J, Webb DM: Rapid evolution of primate antiviral enzyme APOBEC3G. Hum Mol Genet 2004, 13:1785-1791.

48. Wedekind JE, Dance GS, Sowden MP, Smith HC: Messenger RNA editing in mammals: new members of the APOBEC family seeking roles in the family business. Trends Genet 2003, 19:207-216.

49. Do H, Vasilescu A, Diop G, Hirtzig T, Heath SC, Coulonges C, Rappaport J, Therwath A, Lathrop M, Matsuda F, Zagury JF: Exhaustive genotyping of the CEM15 (APOBEC3G) gene and absence of association with AIDS progression in a French cohort. J Infect Dis 2005, 191:159-163.

50. Sawyer SL, Emerman M, Malik HS: Ancient adaptive evolution of the primate antiviral DNA-editing enzyme APOBEC3G. PLOS Biol 2004, 2: E275.

51. Bizinoto MC, Leal E, Diaz RS, Janini LM: Loci polymorphisms of the APOBEC3G gene in HIV type 1-infected Brazilians. AIDS Res Hum Retroviruses 2011, 27:137-141.

52. An P, Bleiber G, Duggal P, Nelson G, May M, Mangeat B, Alobwede I, Trono $D$, Vlahov D, Donfield S, et al: APOBEC3G genetic variants and their influence on the progression to AIDS. J Virol 2004, 78:11070-11076.

53. Kieffer TL, Kwon P, Nettles RE, Han Y, Ray SC, Siliciano RF: G->A hypermutation in protease and reverse transcriptase regions of human immunodeficiency virus type 1 residing in resting CD4+ T cells in vivo. $J$ Virol 2005, 79:1975-1980.

54. Pace C, Keller J, Nolan D, James I, Gaudieri S, Moore C, Mallal S: Population level analysis of human immunodeficiency virus type 1 hypermutation and its relationship with APOBEC3G and vif genetic variation. J Virol 2006, 80:9259-9269

55. Piantadosi A, Humes D, Chohan B, McClelland RS, Overbaugh J: Analysis of the percentage of human immunodeficiency virus type 1 sequences that are hypermutated and markers of disease progression in a longitudinal cohort, including one individual with a partially defective Vif. J Virol 2009, 83:7805-7814

56. Land AM, Ball TB, Luo M, Pilon R, Sandstrom P, Embree JE, Wachihi C, Kimani J, Plummer FA: Human immunodeficiency virus (HIV) type 1 proviral hypermutation correlates with CD4 count in HIV-infected women from Kenya. J Virol 2008, 82:8172-8182

57. Gandhi SK, Siliciano JD, Bailey JR, Siliciano RF, Blankson JN: Role of APOBEC3G/ F-mediated hypermutation in the control of human immunodeficiency virus type 1 in elite suppressors. J Virol 2008, 82:3125-3130.

58. Preston BD, Poiesz BJ, Loeb LA: Fidelity of HIV-1 reverse transcriptase. Science 1988, 242:1168-1171.
59. Yu X, Yu Y, Liu B, Luo K, Kong W, Mao P, Yu XF: Induction of APOBEC3G ubiquitination and degradation by an HIV-1 Vif-Cul5-SCF complex. Science 2003, 302:1056-1060.

60. Santa-Marta M, da Silva FA, Fonseca AM, Goncalves J: HIV-1 Vif can directly inhibit apolipoprotein B mRNA-editing enzyme catalytic polypeptide-like $3 \mathrm{G}$-mediated cytidine deamination by using a single amino acid interaction and without protein degradation. J Biol Chem 2005, 280:8765-8775.

61. Khan MA, Aberham C, Kao S, Akari H, Gorelick R, Bour S, Strebel K: Human immunodeficiency virus type $1 \mathrm{Vif}$ protein is packaged into the nucleoprotein complex through an interaction with viral genomic RNA. J Virol 2001, 75:7252-7265.

62. Goncalves J, Santa-Marta M: HIV-1 Vif and APOBEC3G: multiple roads to one goal. Retrovirology 2004, 1:28.

63. Sheehy AM, Gaddis NC, Malim MH: The antiretroviral enzyme APOBEC3G is degraded by the proteasome in response to HIV-1 Vif. Nat Med 2003, 9:1404-1407.

64. Mehle A, Strack B, Ancuta P, Zhang C, McPike M, Gabuzda D: Vif overcomes the innate antiviral activity of APOBEC3G by promoting its degradation in the ubiquitin-proteasome pathway. J Biol Chem 2004, 279:7792-7798.

65. Goila-Gaur R, Strebel K: HIV-1 Vif, APOBEC, and intrinsic immunity. Retrovirology 2008, 5:51

66. Stopak K, de Noronha C, Yonemoto W, Greene WC: HIV-1 Vif blocks the antiviral activity of APOBEC3G by impairing both its translation and intracellular stability. Mol Cell 2003, 12:591-601.

67. Strebel K, Luban J, Jeang KT: Human cellular restriction factors that target HIV-1 replication. BMC Med 2009, 7:48.

68. Conticello SG, Harris RS, Neuberger MS: The Vif protein of HIV triggers degradation of the human antiretroviral DNA deaminase APOBEC3G. Curr Biol 2003, 13:2009-2013.

69. Kao S, Akari H, Khan MA, Dettenhofer M, Yu XF, Strebel K: Human immunodeficiency virus type $1 \mathrm{Vif}$ is efficiently packaged into virions during productive but not chronic infection. J Virol 2003, 77:1131-1140.

70. Soros VB, Greene WC: APOBEC3G and HIV-1: strike and counterstrike. Curr Infect Dis Rep 2006, 8:317-323.

71. Svarovskaia ES, Xu H, Mbisa JL, Barr R, Gorelick RJ, Ono A, Freed EO, Hu WS, Pathak VK: Human apolipoprotein B mRNA-editing enzyme-catalytic polypeptide-like $3 \mathrm{G}$ (APOBEC3G) is incorporated into HIV-1 virions through interactions with viral and nonviral RNAs. J Biol Chem 2004, 279:35822-35828.

72. Alce TM, Popik W: APOBEC3G is incorporated into virus-like particles by a direct interaction with HIV-1 Gag nucleocapsid protein. J Biol Chem 2004, 279:34083-34086.

73. Ma J, Li X, Xu J, Zhang Q, Liu Z, Jia P, Zhou J, Guo F, You X, Yu L, et al: The cellular source for APOBEC3G's incorporation into HIV-1. Retrovirology 2011, 8:2.

74. Mariani R, Chen D, Schrofelbauer B, Navarro F, Konig R, Bollman B, Munk C, Nymark-McMahon H, Landau NR: Species-specific exclusion of APOBEC3G from HIV-1 virions by Vif. Cell 2003, 114:21-31.

75. Wissing S, Galloway NL, Greene WC: HIV-1 Vif versus the APOBEC3 cytidine deaminases: an intracellular duel between pathogen and host restriction factors. Mol Aspects Med 2010, 31:383-397.

76. Xu H, Chertova E, Chen J, Ott DE, Roser JD, Hu WS, Pathak VK Stoichiometry of the antiviral protein APOBEC3G in HIV-1 virions. Virology 2007, 360:247-256

77. Mercenne G, Bernacchi S, Richer D, Bec G, Henriet S, Paillart JC, Marquet R: HIV-1 Vif binds to APOBEC3G mRNA and inhibits its translation. Nucleic Acids Res 2010, 38:633-646.

78. Kao S, Khan MA, Miyagi E, Plishka R, Buckler-White A, Strebel K: The human immunodeficiency virus type 1 Vif protein reduces intracellular expression and inhibits packaging of APOBEC3G (CEM15), a cellular inhibitor of virus infectivity. J Virol 2003, 77:11398-11407.

79. Opi S, Kao S, Goila-Gaur R, Khan MA, Miyagi E, Takeuchi H, Strebel K: Human immunodeficiency virus type 1 Vif inhibits packaging and antiviral activity of a degradation-resistant APOBEC3G variant. J Virol 2007, 81:8236-8246.

80. Kao S, Goila-Gaur R, Miyagi E, Khan MA, Opi S, Takeuchi H, Strebel K: Production of infectious virus and degradation of APOBEC 3G are separable functional properties of human immunodeficiency virus type 1 Vif. Virology 2007, 369:329-339.

81. Jager S, Kim DY, Hultquist JF, Shindo K, LaRue RS, Kwon E, Li M, Anderson BD, Yen L, Stanley D, et al: Vif hijacks CBF-beta to degrade APOBEC3G and promote HIV-1 infection. Nature 2011, 481:371-375. 
82. Zhang W, Du J, Evans SL, Yu Y, Yu XF: T-cell differentiation factor CBF-beta regulates HIV-1 Vif-mediated evasion of host restriction. Nature 2011, 481:376-379.

83. Hamilton CE, Papavasiliou FN, Rosenberg BR: Diverse functions for DNA and RNA editing in the immune system. RNA Biol 2010, 7:220-228.

84. Sadler HA, Stenglein MD, Harris RS, Mansky LM: APOBEC3G contributes to HIV-1 variation through sublethal mutagenesis. J Virol 2010, 84:7396-7404.

85. Goila-Gaur R, Khan MA, Miyagi E, Strebel K: Differential sensitivity of "old" versus "new" APOBEC3G to human immunodeficiency virus type 1 vif. $J$ Virol 2009, 83:1156-1160

86. Sakai K, Dimas J, Lenardo MJ: The Vif and Vpr accessory proteins independently cause HIV-1-induced T cell cytopathicity and cell cycle arrest. Proc Natl Acad Sci U S A 2006, 103:3369-3374.

87. Wang J, Shackelford JM, Casella CR, Shivers DK, Rapaport EL, Liu B, Yu XF, Finkel TH: The Vif accessory protein alters the cell cycle of human immunodeficiency virus type 1 infected cells. Virology 2007, 359: 243-252.

88. Wang J, Shackelford JM, Selliah N, Shivers DK, O'Neill E, Garcia JV, Muthumani K, Weiner D, Yu XF, Gabuzda D, Finkel TH: The HIV-1 Vif protein mediates degradation of $\mathrm{Vpr}$ and reduces $\mathrm{Vpr}$-induced cell cycle arrest. DNA Cell Biol 2008, 27:267-277.

89. DeHart $J$, Bosque A, Harris RS, Planelles V: Human immunodeficiency virus type 1 Vif induces cell cycle delay via recruitment of the same E3 ubiquitin ligase complex that targets APOBEC3 proteins for degradation. J Virol 2008, 82:9265-9272.

90. Chiu YL, Greene WC: APOBEC3G: an intracellular centurion. Philos Trans $R$ Soc Lond B Biol Sci 2009, 364:689-703.

91. Kreisberg JF, Yonemoto W, Greene WC: Endogenous factors enhance HIV infection of tissue naive CD4 T cells by stimulating high molecular mass APOBEC3G complex formation. J Exp Med 2006, 203:865-870.

92. Chiu YL, Greene WC: The APOBEC3 cytidine deaminases: an innate defensive network opposing exogenous retroviruses and endogenous retroelements. Annu Rev Immunol 2008, 26:317-353.

93. Wichroski MJ, Robb GB, Rana TM: Human retroviral host restriction factors APOBEC3G and APOBEC3F localize to MRNA processing bodies. PLOS Pathog 2006, 2:e41.

94. Kozak SL, Marin M, Rose KM, Bystrom C, Kabat D: The anti-HIV-1 editing enzyme APOBEC3G binds HIV-1 RNA and messenger RNAs that shuttle between polysomes and stress granules. J Biol Chem 2006, 281:2910529119.

95. Chiu YL: Biochemical fractionation and purification of high-molecularmass APOBEC3G complexes. Methods Mol Biol 2011, 718:185-206.

96. Gallois-Montbrun S, Kramer B, Swanson CM, Byers H, Lynham S, Ward M, Malim $\mathrm{MH}$ : Antiviral protein APOBEC3G localizes to ribonucleoprotein complexes found in P bodies and stress granules. J Virol 2007, 81:2165-2178.

97. Strebel K, Khan MA: APOBEC3G encapsidation into HIV-1 virions: which RNA is it? Retrovirology 2008, 5:55.

98. Khan MA, Goila-Gaur R, Opi S, Miyagi E, Takeuchi H, Kao S, Strebel K: Analysis of the contribution of cellular and viral RNA to the packaging of APOBEC3G into HIV-1 virions. Retrovirology 2007, 4:48.

99. Khan MA, Kao S, Miyagi E, Takeuchi H, Goila-Gaur R, Opi S, Gipson CL, Parslow TG, Ly H, Strebel K: Viral RNA is required for the association of APOBEC3G with human immunodeficiency virus type 1 nucleoprotein complexes. J Virol 2005, 79:5870-5874.

100. Wang T, Tian C, Zhang W, Luo K, Sarkis PT, Yu L, Liu B, Yu Y, Yu XF: 7SL RNA mediates virion packaging of the antiviral cytidine deaminase APOBEC3G. J Virol 2007, 81:13112-13124.

101. Onafuwa-Nuga AA, Telesnitsky A, King SR: 7SL RNA, but not the 54-kd signal recognition particle protein, is an abundant component of both infectious HIV-1 and minimal virus-like particles. RNA 2006, 12:542-546.

102. Shandilya SM, Nalam MN, Nalivaika EA, Gross PJ, Valesano JC, Shindo K, Li M, Munson M, Royer WE, Harjes E, et al: Crystal structure of the APOBEC3G catalytic domain reveals potential oligomerization interfaces. Structure 2010, 18:28-38.

103. Chen KM, Harjes E, Gross PJ, Fahmy A, Lu Y, Shindo K, Harris RS, Matsuo H: Structure of the DNA deaminase domain of the HIV-1 restriction factor APOBEC3G. Nature 2008, 452:116-119.

104. Holden LG, Prochnow C, Chang YP, Bransteitter R, Chelico L, Sen U, Stevens RC, Goodman MF, Chen XS: Crystal structure of the anti-viral APOBEC3G catalytic domain and functional implications. Nature 2008, 456:121-124.
105. Huthoff H, Autore F, Gallois-Montbrun S, Fraternali F, Malim MH: RNAdependent oligomerization of APOBEC3G is required for restriction of HIV-1. PLoS Pathog 2009, 5:e1000330.

106. Wang X, Dolan PT, Dang Y, Zheng YH: Biochemical differentiation of APOBEC3F and APOBEC3G proteins associated with HIV-1 life cycle. J Biol Chem 2007, 282:1585-1594.

107. Kamata M, Nagaoka Y, Chen IS: Reassessing the role of APOBEC3G in human immunodeficiency virus type 1 infection of quiescent CD4+ Tcells. PLOS Pathog 2009, 5:e1000342.

108. de Sio FR Santoni, Trono D: APOBEC3G-depleted resting CD4+ T cells remain refractory to HIV1 infection. PLoS One 2009, 4:6571.

109. Goila-Gaur R, Khan MA, Miyagi E, Kao S, Opi S, Takeuchi H, Strebel K: HIV-1 Vif promotes the formation of high molecular mass APOBEC3G complexes. Virology 2008, 372:136-146.

110. Johnson VA, Calvez V, Gunthard HF, Paredes R, Pillay D, Shafer R, Wensing AM, Richman DD: 2011 update of the drug resistance mutations in HIV-1. Top Antivir Med 2011, 19:156-164.

111. Berkhout $B$, de Ronde $A$ : APOBEC3G versus reverse transcriptase in the generation of HIV-1 drug-resistance mutations. AIDS 2004, 18:1861-1863.

112. Jern P, Russell RA, Pathak VK, Coffin JM: Likely role of APOBEC3G-mediated G-to-A mutations in HIV-1 evolution and drug resistance. PLOS Pathog 2009, 5:e1000367.

113. Suspene R, Rusniok C, Vartanian JP, Wain-Hobson S: Twin gradients in APOBEC3 edited HIV-1 DNA reflect the dynamics of lentiviral replication. Nucleic Acids Res 2006, 34:4677-4684.

114. Mulder LC, Harari A, Simon V: Cytidine deamination induced HIV-1 drug resistance. Proc Natl Acad Sci U S A 2008, 105:5501-5506.

115. Dang $Y$, Wang $X$, Zhou T, York IA, Zheng $Y H$ : Identification of a novel WxSLVK motif in the $\mathrm{N}$ terminus of human immunodeficiency virus and simian immunodeficiency virus Vif that is critical for APOBEC3G and APOBEC3F neutralization. J Virol 2009, 83:8544-8552.

116. Borrow P, Lewicki H, Hahn BH, Shaw GM, Oldstone MB: Virus-specific CD8+ cytotoxic T-lymphocyte activity associated with control of viremia in primary human immunodeficiency virus type 1 infection. J Virol 1994, 68:6103-6110.

117. Rinaldo C, Huang XL, Fan ZF, Ding M, Beltz L, Logar A, Panicali D, Mazzara G, Liebmann J, Cottrill M, et al: High levels of anti-human immunodeficiency virus type 1 (HIV-1) memory cytotoxic T-lymphocyte activity and low viral load are associated with lack of disease in HIV-1-infected long-term nonprogressors. J Virol 1995, 69:5838-5842.

118. Koup RA, Safrit JT, Cao Y, Andrews CA, McLeod G, Borkowsky W, Farthing C, Ho DD: Temporal association of cellular immune responses with the initial control of viremia in primary human immunodeficiency virus type 1 syndrome. J Virol 1994, 68:4650-4655.

119. Duffy S, Shackelton LA, Holmes EC: Rates of evolutionary change in viruses: patterns and determinants. Nat Rev Genet 2008, 9:267-276.

120. Domingo $E$, Holland JJ: RNA virus mutations and fitness for survival. Annu Rev Microbiol 1997, 51:151-178.

121. Frost SD, Nijhuis M, Schuurman R, Boucher CA, Brown AJ: Evolution of lamivudine resistance in human immunodeficiency virus type 1-infected individuals: the relative roles of drift and selection. J Virol 2000, 74:6262-6268.

122. Moya A, Holmes EC, Gonzalez-Candelas F: The population genetics and evolutionary epidemiology of RNA viruses. Nat Rev Microbiol 2004, 2:279-288.

123. Kim EY, Bhattacharya T, Kunstman K, Swantek P, Koning FA, Malim MH, Wolinsky SM: Human APOBEC3G-mediated editing can promote HIV-1 sequence diversification and accelerate adaptation to selective pressure. J Virol 2010, 84:10402-10405.

124. Yebra G, Holguin A: Mutation Vif-22 $\mathrm{H}$, which allows HIV-1 to use the APOBEC3G hypermutation to develop resistance, could appear more quickly in certain non-B variants. J Antimicrob Chemother 2011, 66:941-942.

125. Fourati S, Malet I, Binka M, Boukobza S, Wirden M, Sayon S, Simon A, Katlama C, Simon V, Calvez V, Marcelin AG: Partially active HIV-1 Vif alleles facilitate viral escape from specific antiretrovirals. AIDS 2010, 24:2313-2321.

126. Harrer T, Harrer E, Kalams SA, Elbeik T, Staprans SI, Feinberg MB, Cao Y, Ho DD, Yilma T, Caliendo AM, et al: Strong cytotoxic T cell and weak neutralizing antibody responses in a subset of persons with stable nonprogressing HIV type 1 infection. AIDS Res Hum Retroviruses 1996, 12:585-592.

127. Shankar $P$, Russo M, Harnisch B, Patterson M, Skolnik $P$, Lieberman J: Impaired function of circulating HIV-specific CD8(+) T cells in chronic human immunodeficiency virus infection. Blood 2000, 96:3094-3101. 
128. Heintel T, Sester M, Rodriguez MM, Krieg C, Sester U, Wagner R, Pees HW, Gartner B, Maier R, Meyerhans A: The fraction of perforin-expressing HIV-specific CD8 T cells is a marker for disease progression in HIV infection. AIDS 2002, 16:1497-1501.

129. Ogg GS, Jin X, Bonhoeffer S, Dunbar PR, Nowak MA, Monard S, Segal JP, Cao Y, Rowland-Jones SL, Cerundolo V, et al: Quantitation of HIV-1-specific cytotoxic T lymphocytes and plasma load of viral RNA. Science 1998, 279:2103-2106.

130. Yusim K, Kesmir C, Gaschen B, Addo MM, Altfeld M, Brunak S, Chigaev A, Detours V, Korber BT: Clustering patterns of cytotoxic T-lymphocyte epitopes in human immunodeficiency virus type 1 (HIV-1) proteins reveal imprints of immune evasion on HIV-1 global variation. J Virol 2002, 76:8757-8768

131. Jones NA, Wei X, Flower DR, Wong M, Michor F, Saag MS, Hahn BH, Nowak MA, Shaw GM, Borrow P: Determinants of human immunodeficiency virus type 1 escape from the primary CD8+ cytotoxic T lymphocyte response. $J$ Exp Med 2004, 200:1243-1256.

132. Tomiyama H, Fujiwara M, Oka S, Takiguchi M: Cutting Edge: Epitope-dependent effect of Nef-mediated HLA class I down-regulation on ability of HIV-1-specific CTLs to suppress HIV-1 replication. J Immunol 2005, 174:36-40.

133. Barouch $\mathrm{DH}$, Letvin NL: Viral evolution and challenges in the development of HIV vaccines. Vaccine 2002, 20(Suppl 4):A66-A68

134. Borrow $P$, Lewicki $H$, Wei X, Horwitz MS, Peffer N, Meyers $H$, Nelson JA, Gairin JE, Hahn BH, Oldstone MB, Shaw GM: Antiviral pressure exerted by HIV-1-specific cytotoxic T lymphocytes (CTLs) during primary infection demonstrated by rapid selection of CTL escape virus. Nat Med 1997, 3:205-211.

135. Geels MJ, Cornelissen M, Schuitemaker H, Anderson K, Kwa D, Maas J, Dekker JT, Baan E, Zorgdrager F, van den Burg R, et al: Identification of sequential viral escape mutants associated with altered T-cell responses in a human immunodeficiency virus type 1-infected individual. J Virol $2003,77: 12430-12440$

136. Wood N, Bhattacharya T, Keele BF, Giorgi E, Liu M, Gaschen B, Daniels M, Ferrari G, Haynes BF, McMichael A, et al: HIV evolution in early infection: selection pressures, patterns of insertion and deletion, and the impact of APOBEC. PLoS Pathog 2009, 5:e1000414.

137. Casartelli N, Guivel-Benhassine F, Bouziat R, Brandler S, Schwartz O, Moris A: The antiviral factor APOBEC3G improves CTL recognition of cultured HIV-infected T cells. J Exp Med 2010, 207:39-49.

138. Sakuma R, Noser JA, Ohmine S, Ikeda Y: Inhibition of HIV-1 replication by simian restriction factors, TRIM5alpha and APOBEC3G. Gene Ther 2007, 14:185-189

139. Cen S, Peng ZG, Li XY, Li ZR, Ma J, Wang YM, Fan B, You XF, Wang YP, Liu F, et al: Small molecular compounds inhibit HIV-1 replication through specifically stabilizing APOBEC3G. J Biol Chem 2010, 285:16546-16552.

140. Chen H, Wang LW, Huang YQ, Gong ZJ: Interferon-alpha Induces High Expression of APOBEC3G and STAT-1 in Vitro and in Vivo. Int J Mol Sci 2010, 11:3501-3512

141. Pillai SK, Wong JK, Barbour JD: Turning up the volume on mutational pressure: is more of a good thing always better? (A case study of HIV-1 Vif and APOBEC3). Retrovirology 2008, 5:26.

142. Vazquez-Perez JA, Ormsby CE, Hernandez-Juan R, Torres KJ, Reyes-Teran G: APOBEC3G mRNA expression in exposed seronegative and early stage HIV infected individuals decreases with removal of exposure and with disease progression. Retrovirology 2009, 6:23

143. Jin X, Brooks A, Chen H, Bennett R, Reichman R, Smith H: APOBEC3G/ CEM15 (hA3G) mRNA levels associate inversely with human immunodeficiency virus viremia. J Virol 2005, 79:11513-11516.

144. Cho SJ, Drechsler H, Burke RC, Arens MQ, Powderly W, Davidson NO: APOBEC3F and APOBEC3G mRNA levels do not correlate with human immunodeficiency virus type 1 plasma viremia or CD4+ T-cell count. $J$ Virol 2006, 80:2069-2072

145. Mussil B, Sauermann U, Motzkus D, Stahl-Hennig C, Sopper S: Increased APOBEC 3G and APOBEC3F expression is associated with low viral load and prolonged survival in simian immunodeficiency virus infected rhesus monkeys. Retrovirology 2011, 8:77.

146. Russell RA, Moore MD, Hu WS, Pathak VK: APOBEC3G induces a hypermutation gradient: purifying selection at multiple steps during HIV-1 replication results in levels of G-to-A mutations that are high in
DNA, intermediate in cellular viral RNA, and low in virion RNA. Retrovirology 2009, 6:16.

147. Hu WS, Temin HM: Retroviral recombination and reverse transcription. Science 1990, 250:1227-1233.

148. Zhang J, Temin HM: Rate and mechanism of nonhomologous recombination during a single cycle of retroviral replication. Science 1993, 259:234-238.

149. Temin HM: Mixed infection with two types of Rous sarcoma virus. Virology 1961, 13:158-163.

150. Holland JJ, De La Torre JC, Steinhauer DA: RNA virus populations as quasispecies. Curr Top Microbiol Immunol 1992, 176:1-20.

151. Mansky LM, Temin HM: Lower in vivo mutation rate of human immunodeficiency virus type 1 than that predicted from the fidelity of purified reverse transcriptase. J Virol 1995, 69:5087-5094.

152. Coffin JM: Genetic diversity and evolution of retroviruses. Curr Top Microbiol Immunol 1992, 176:143-164.

153. Katz RA, Skalka AM: Generation of diversity in retroviruses. Annu Rev Genet 1990, 24:409-445.

154. Jetzt $A E$, Y $u$ H, Klarmann GJ, Ron Y, Preston BD, Dougherty JP: High rate of recombination throughout the human immunodeficiency virus type 1 genome. J Virol 2000, 74:1234-1240.

155. Charpentier C, Nora T, Tenaillon O, Clavel F, Hance AJ: Extensive recombination among human immunodeficiency virus type 1 quasispecies makes an important contribution to viral diversity in individual patients. J Virol 2006, 80:2472-2482.

156. Chen J, Powell D, Hu WS: High frequency of genetic recombination is a common feature of primate lentivirus replication. J Virol 2006, 80: 9651-9658.

157. Onafuwa-Nuga A, Telesnitsky A: The remarkable frequency of human immunodeficiency virus type 1 genetic recombination. Microbiol Mol Biol Rev 2009, 73:451-480. Table of Contents.

158. Jung A, Maier R, Vartanian JP, Bocharov G, Jung V, Fischer U, Meese E, Wain-Hobson S, Meyerhans A: Recombination: Multiply infected spleen cells in HIV patients. Nature 2002, 418:144

159. Nikolenko GN, Svarovskaia ES, Delviks KA, Pathak VK: Antiretroviral drug resistance mutations in human immunodeficiency virus type 1 reverse transcriptase increase template-switching frequency. J Virol 2004, 78:8761-8770

160. Malim MH, Emerman M: HIV-1 sequence variation: drift, shift, and attenuation. Cell 2001, 104:469-472.

161. van Hemert FJ, Berkhout B: The tendency of lentiviral open reading frames to become A-rich: constraints imposed by viral genome organization and cellular tRNA availability. J Mol Evol 1995, 41:132-140.

162. Berkhout B, van Hemert FJ: The unusual nucleotide content of the HIV RNA genome results in a biased amino acid composition of HIV proteins. Nucleic Acids Res 1994, 22:1705-1711.

163. Berkhout B, Das AT, Beerens N: HIV-1 RNA editing, hypermutation, and error-prone reverse transcription. Science 2001, 292:7.

164. Chelico L, Pham P, Goodman MF: Mechanisms of APOBEC3G-catalyzed processive deamination of deoxycytidine on single-stranded DNA. Nat Struct Mol Biol 2009, 16:454-455. author reply 455-456.

doi:10.1186/1742-4690-9-35

Cite this article as: Monajemi et al:: Emerging complexities of APOBEC3G action on immunity and viral fitness during HIV infection and treatment. Retrovirology 2012 9:35 